\title{
Primary Adult Retroperitoneal Sarcoma: A Comprehensive Genomic Profiling Study
}

\author{
Andrea Necchi, ${ }^{1}$ Giuseppe Basile, $₫ 2$ Filippo Pederzoli², Marco Bandini, ${ }^{2}$ Petros Grivas, ${ }^{3}$ \\ Gennady Bratslavsky, ${ }^{4}$ Philippe E. Spiess, ${ }^{5}$ J. Keith Killian, ${ }^{6}$ Douglas I. Lin, ${ }^{6}$ Erik Williams, ${ }^{6}$ \\ Shakti Ramkissoon, ${ }^{6}$ Eric A. Severson, ${ }^{6}$ Brian M. Alexander, ${ }^{6}$ Jeffrey Venstrom, $^{6}$ \\ Prasanth Reddy, ${ }^{6}$ Kimberly McGregor, ${ }^{6}$ Julia A. Elvin, ${ }^{6}$ Alexa B. Schrock, ${ }^{6}$ Dean C. Pavlick, ${ }^{6}$ \\ Dexter X. Jin, ${ }^{6}$ Sally E. Trabucco, ${ }^{6}$ Natalie Danziger, ${ }^{6}$ Jeffrey S. Ross ${ }^{5,6}$ \\ 'Department of Medical Oncology, IRCCS Ospedale San Raffaele, Vita-Salute San Raffaele University, Milan, Italy ${ }^{2}$ Urological Research Institute (URI), Unit of Urology, \\ IRCCS Ospedale San Raffaele, Vita-Salute San Raffaele University, Milan, Italy ${ }^{3}$ University of Washington, Fred Hutchinson Cancer Research Center, Seattle Cancer \\ Care Alliance, United States ${ }^{4}$ SUNY Upstate Medical University, Syracuse, United States ${ }^{5}$ Moffitt Cancer Center and Research Institute, Tampa, United States \\ ${ }^{6}$ Foundation Medicine Inc., Cambridge, United States
}

\section{Abstract}

Background Adult primary retroperitoneal sarcomas (RPSs) are a group of heterogeneous tumors with different histological subtypes. Comprehensive genomic profiling (CGP) analyses have recently provided significant insights into the biology of sarcomas by identifying genomic alterations (GAs) which could benefit from targeted therapies.

Methods RPS were evaluated by CGP using next-generation sequencing of up to 406 cancer-related genes. Tumor mutational burden (TMB) was determined on 0.83 to 1.14 mut/Mb of sequenced DNA. Finally, PD-L1 expression was determined.

Results Overall, 296 cases of primary RPS were analyzed. Liposarcoma (LPS) subtype had more GA/tumor than leiomyosarcoma (LMS) subtypes, with follicular dendritic cell sarcomas harboring the highest and synovial sarcomas the lowest. TP53 and Rb1 alterations were the highest in LMS, and CDK4/6 and MDM2 in LPS. However, both the TMB and targetable GA rates were low across subtypes. PD-L1 immunostaining was low positive in $21 \%$ and high positive in $5 \%$ of patients, respectively.

Conclusions CGP analysis revealed that potentially actionable genomic targets were rare in our cohort of RPS. Moreover, RPSs seem less likely to respond to immune checkpoint inhibitors based on putative biomarkers status. Nevertheless, genomic stratification according to histological subtypes led to description of GAs that can inform future clinical trials design.

\section{Key Words}

Retroperitoneal sarcoma, comprehensive genomic profiling, targeted therapy

\section{Competing Interests}

Dr P Grivas (all unrelated in the last 3 years): consulting: AstraZeneca; Bayer; Bristol Myers Squibb; Clovis Oncology; Dyania Health, Driver; EMD Serono; Exelixis; Foundation Medicine: Genentech/Roche; Genzyme; GlaxoSmithKline; Heron Therapeutics; Immunomedics, Janssen; Merck; Mirati Therapeutics; Pfizer; Seattle Genetics; OED Therapeutics. Research Funding to Institution: Merck; Pfizer, Clovis Oncology, Bavarian Nordic, Immunomedics, Debiopharm, Bristol Myers Squibb, OED Therapeutics, GlaxoSmithKline, Kure It Cancer Research. Dr A Necchi: consultant and advisor, Merck, Roche, Astra Zeneca, Janssen, Clovis Oncology, Incyte, BioClin Therapeutics; Bayer, Bristol Myers Squibb; Research grants (Institution): Merck, Astra Zeneca.

\section{Article Information}

Received on March 28, 2021

Accepted on May 15, 2021

Soc Int Urol J. 2021;2(4):216-228

DOI: 10.48083/VOGF2319 


\section{Abbreviations}

CGP comprehensive genomic profiling

FDCS follicular dendritic cell sarcoma

GA genomic alteration

ICl immune checkpoint inhibitor

LMS leiomyosarcoma

LPS liposarcoma

MPNST malignant peripheral nerve sheath tumors

MSI microsatellite instability

OS overall survival

PFS progression-free survival

PLS pleomorphic sarcoma

PRS primary retroperitoneal sarcoma

SFT solitary fibrous tumors

SS synovial sarcomas

TMB tumor mutational burden

TKI tyrosine kinase inhibitor

\section{Introduction}

Adult primary retroperitoneal sarcomas (RPSs) are rare malignancies that encompass a variety of clinical and pathological entities, with distinct histology and cancer biology[1]. The reported yearly crude incidence rate of soft-tissue sarcomas of the retroperitoneum and peritoneum is 0.31 per 100000 individuals in Europe, with a 5-year relative survival rate of $38.8 \%$ [2]. RPSs are usually classified according to the normal mesenchymal tissue they most closely resemble. The correct identification of the histological subtype constitutes a mainstay in the multidisciplinary management of RPS, as different entities are more or less responsive to systemic therapy and/or radiation, thus influencing the therapeutic plan $[3,4]$. Nevertheless, together with traditional histology-based classification of sarcomas, novel data about the genomic, epigenetic, and immunological landscape of these rare malignancies are emerging to potentially guide better stratification. Particularly, sarcomas have been traditionally grouped into 2 broad categories based on genomic alterations: sarcomas with simple karyotypes harboring distinct alterations, such as reciprocal chromosomal translocations and specific oncogenic mutations, and those with more complex, unbalanced karyotypes[5]. However, this crude dichotomy does not account for the complex heterogeneity within a given histology and between different subtypes, highlighting the need for a widespread implementation of molecular profiles in sarcomas.

In this context, comprehensive genomic profiling (CGP) analysis can provide significant insights into the biology of several tumors, allowing detection of numerous genomic alterations that could help elucidate the biology and potentially suggest strategies for precision oncology clinical trials [6,7]. In this study, we profiled a group of 296 RPS and analyzed the frequency of genomic alterations (GAs), hypothesizing that we would identify distinct therapeutic opportunities for patients affected by these rare malignancies.

\section{Methods}

Approval for this study was obtained from the Western Institutional Review Board (Protocol No. 20152817). A retrospective database search of a Clinical Laboratory Improvement Amendments certified, and College of American Pathologists accredited reference molecular laboratory was performed for all available primary RPS cases. The cases were previously assayed by CGP via both DNA- and RNA-based targeted next-generation sequencing (Foundation Medicine, Cambridge, MA) during the course of standard clinical care at other institutions. Clinicopathological data, including patient age and gender, routine histology and immunohistochemical staining results, and confirmation that the sarcomas were primary in the retroperitoneum and not metastases from other nonretroperitoneal primary sarcomas, were extracted from clinicopathology reports. The pathologic diagnosis of primary RPS and associated morphological features were centrally re-evaluated on routine $H \& E$ slides of tissue sections submitted for genomic profiling. Particularly, all cases included in this study were evaluated by an experienced board-certified pathologist at the time of specimen arrival in the laboratory, and then reviewed by a single pathologist to confirm the diagnosis and origin in the retroperitoneum.

All samples forwarded for DNA and RNA extraction contained a minimum of $20 \%$ tumor cells. The samples were assayed using next-generation sequencing for all coding exons from at least 406 cancer-related genes, plus additional select introns from up to 31 genes frequently rearranged in cancer. Patient samples were sequenced and evaluated for genomic alterations including base substitutions, insertions, deletions, copy number alterations (amplifications and homozygous deletions), and gene fusions/rearrangements, as previously described[8,9]. RNA-sequencing of 265 genes was performed for rearrangement analysis. The bioinformatics processes used in this study included Bayesian algorithms to detect base substitutions, local assembly algorithms to detect short insertions and deletions, a comparison with process-matched normal control samples to detect gene copy number alterations, and an analysis of chimeric read pairs to identify gene fusions as previously described $[8,9]$. To visualize the sequencing data results, an OncoPrint plot was 
generated with the online tools as described by Gao et al.[10] and Cerami et al.[11].

Tumor mutational burden (TMB) was determined on 0.83 to $1.14 \mathrm{Mb}$ of sequenced DNA using an algorithm, as previously described[12]. In this study, low TMB scores were defined as $<6$ mut/Mb, intermediate TMB as 6 to $19 \mathrm{mut} / \mathrm{Mb}$, and high $\mathrm{TMB}$ as $\geq 20 \mathrm{mut} / \mathrm{Mb}$. The TMB cut-offs used in this study were the levels that had been in use prior to the U.S. Food and Drug Administration (FDA) approval of pembrolizumab in solid tumors featuring a TMB $>10 \mathrm{mut} / \mathrm{Mb}$. Assessment of microsatellite instability (MSI) was performed from DNA sequencing across 114 significant loci[13]. Each microsatellite locus had repeat length of 7 to $39 \mathrm{bp}$. The next-generation sequencing-based microsatellite instability score was translated into categorical MSI high, MSI intermediate, or microsatellite stable tumors by unsupervised clustering of specimens for which microsatellite instability status was previously assessed via gold standard methods[13]. PD-L1 expression was determined on subsets of the tumors using the DAKO $22 \mathrm{C} 3$ assay with low positive tumor cell scoring defined as $1 \%$ to $49 \%$ staining and high positive tumor cell scoring defined as $\geq 50 \%$ staining. The cut-offs for the Dako 22C3 staining are those currently being used in the United States for the selection of patients with nonsmall cell lung cancer for treatment with single agent pembrolizumab.

\section{Results}

The clinical and genomic features of the 296 cases of primary RPS are shown in Table 1 . All cases were clinically advanced and frequently resistant to the most recent therapy the patient had received at the time sequencing was ordered. There were 155 liposarcomas (LPS), 74 leiomyosarcomas (LMS), 44 pleomorphic sarcomas (PLS), 7 solitary fibrous tumors (SFT), 6 malignant peripheral nerve sheath tumors (MPNST), 5 synovial sarcomas (SS), and 5 follicular dendritic cell sarcomas (FDCS). Three cases of fibrosarcoma/ fibromyxoid sarcoma were included in the PLS group. OncoPrint plots of the most frequent GAs recorded in the overall cohort and each subtype is reported in Supplementary material 1.

The median age of all patients was 59 years, similar in all groups except for MPNST and SS, with significantly younger patients. The number of GAs per tumor was similar across the overall cohort and ranged from 5.1 to 7.4. LMS and SS subtypes exhibited the lowest GA/ tumor, while FDCS had the highest (7.4 GA/tumor).

The GAs associated with the RPS as a whole and in the 7 individual RPS subtypes are shown in the longtail plots reported in Figure 1. Alterations in genes not currently linked to possible targeted therapies were identified. TP53 inactivation was frequently reported in LMS and rarely identified in LPS, SS or FDCS, while $R b 1$ inactivating GA was essentially restricted to LMS. Moreover, MDM2 amplification was clearly linked to LPS subtype, while FRS2 amplification, identified in 46\% of all RPS cases, was predominantly associated with the LPS and PLS tumor types.

Alterations potentially linked to targeted therapy selection were identified throughout the RPS cases in limited frequencies. Examples included inactivating NF1/NF2 GA in MPNST, PIK3CA activating mutations and PTEN inactivating mutations and deletions. Moreover, potentially impacting the evaluation of cell cycle inhibitors were the high frequencies of CDK4/6 amplifications, mostly restricted to LPS and PLS, and the $C D K N 2 A / B$ loss identified in PLS, and relatively frequently in MPNST. It should be noted that MTAP loss, which is nearly restricted to tumors with $C D K N 2 A / B$ loss and potentially associated with potential targeted therapies focused on tumor cells arginine metabolism, was not tested for in the current study. Tumor-defining gene fusions included the HMGA2 fusions for the LPS and PLS groups, the STAT6 fusions in SFT type, and SS18 fusions in the SS cases. Rare gene fusions that activate targetable gene kinase domains included very rare detection of $A L K, N T R K$, and ROS1 fusions, all identified at $1 \%$ of LPS and PLS, $2 \%$ of PLS and in 1 out of 6 cases of MPNST.

Biomarkers currently associated with response to immune checkpoint inhibitors (ICIs) were also evaluated. No tumor featured MSI high status. TMB was low throughout this group of tumors, with MPNST having the highest median TMB at 4.8 mut/Mb and SS, SFT, and FDCS all having a median TMB of $<1$ mut/Mb. Low tumor cell PD-L1 expression $(<49 \%)$ was detected in $21 \%$ of RPS cases, with PLS having the highest frequency at 33\%, and SFT, MPNST, SS, and FDCS all having no low positive cases. High positive staining $(\geq$ $50 \%$ ) was present in only $5 \%$ of our cohort and mostly identified in PLS (16\%) and FDCS (20\%) subtypes. Boxplot of TMB analysis of all RPS included and each subtype is reported in Supplementary material 2.

Case examples of genomically profiled RPS are shown in Figures 2 and 3. In Figure 2, a PLS in a 77-year-old woman featured an activating fusion in the NTRK 3 gene with the STRN3 gene [5'-STRN3(ex1-3 NM_014574)(B)NTRK3(ex12-19 NM_002530)]. In Figure 3, a welldifferentiated retroperitoneal LPS showed significant amplification of the CDK4 gene, which has potential to drive therapy selection using specific CDK4 inhibitors in clinical trials. Figure 4 shows a retroperitoneal dedifferentiated liposarcoma which presented with pulmonary metastases and was found to contain an MDM2 amplification and an HMGA2-TSFM fusion. This 
TABLE 1.

Clinical and genomic features in adult retroperitoneal sarcomas

\begin{tabular}{|c|c|c|c|c|c|c|c|c|}
\hline & All Cases & LPS & LMS & PLS & SFT & MPNST & SS & FDCS \\
\hline Number of cases, $n$ & 296 & 155 & 74 & 44 & 7 & 6 & 5 & 5 \\
\hline Female gender, \% & 53 & 42 & 78 & 49 & 57 & 33 & 60 & 60 \\
\hline Median age in years, range & $\begin{array}{c}59 \\
(20-88)\end{array}$ & $\begin{array}{c}60 \\
(29-88)\end{array}$ & $\begin{array}{c}60 \\
(31-86)\end{array}$ & $\begin{array}{c}57 \\
(20-85)\end{array}$ & $\begin{array}{c}52 \\
(31-71)\end{array}$ & $\begin{array}{c}28 \\
(20-53)\end{array}$ & $\begin{array}{c}39 \\
(22-46)\end{array}$ & $\begin{array}{c}56 \\
(30-71)\end{array}$ \\
\hline GA/tumor & 5.1 & 6 & 3.4 & 5.2 & 6 & 5.7 & 2.6 & 7.4 \\
\hline $\begin{array}{l}\text { TP53 Inactivating } \\
\text { SV mutation, \% }\end{array}$ & 24 & 5 & 66 & 26 & 29 & 33 & 0 & 0 \\
\hline $\begin{array}{l}\text { RB1 Inactivating } \\
\text { SV mutation, \% }\end{array}$ & 10 & 1 & 32 & 5 & 0 & 0 & 0 & 0 \\
\hline FRS2 Amplification & 46 & 78 & 0 & 28 & 14 & 0 & 0 & 0 \\
\hline $\begin{array}{l}\text { NF1/NF2 Inactivating } \\
\text { SV mutation, \% }\end{array}$ & 4 & 1 & 1 & 4 & 0 & 83 & 0 & 0 \\
\hline $\begin{array}{l}\text { PIK3CA Activating } \\
\text { SV mutations and } \\
\text { amplifications, \% }\end{array}$ & 3 & 2 & 4 & 0 & 0 & 17 & 0 & 0 \\
\hline $\begin{array}{l}\text { ESR1 Inactivating } \\
\text { SV mutation, \% }\end{array}$ & 7 & 12 & 0 & 0 & 14 & 0 & 0 & 0 \\
\hline CDKN2A Deletion, \% & $<1$ & $<1$ & 1 & 15 & 0 & 83 & 0 & 0 \\
\hline CDKN2B Deletion, \% & $<1$ & $<1$ & 1 & 11 & 0 & 83 & 0 & 0 \\
\hline CDK4/6 Amplification, \% & 52 & 89 & 0 & 28 & 14 & 0 & 0 & 20 \\
\hline $\begin{array}{l}\text { PTEN Deletion/ inactivating } \\
\text { SV mutation, \% }\end{array}$ & 4 & 2 & 12 & 9 & 0 & 0 & 0 & 20 \\
\hline MDM2 Amplification, \% & 54 & 91 & 1 & 30 & 14 & 17 & 0 & 20 \\
\hline $\begin{array}{l}\text { ALK Kinase activating } \\
\text { fusions, } \%\end{array}$ & $<1$ & 1 & 0 & 0 & 0 & 0 & 0 & 0 \\
\hline $\begin{array}{l}\text { ROS1 Kinase activating } \\
\text { fusions, \% }\end{array}$ & $<1$ & 1 & 1 & 0 & 0 & 0 & 0 & 0 \\
\hline $\begin{array}{l}\text { NTRK1-3 Kinase activating } \\
\text { fusions, \% }\end{array}$ & 1 & 1 & 1 & 2 & 0 & 17 & 0 & 0 \\
\hline STAT6 Fusions, \% & 2 & 0 & 0 & 0 & 86 & 0 & 0 & 0 \\
\hline HMGA2 Fusions, \% & 17 & 28 & 1 & 11 & 0 & 0 & 0 & 0 \\
\hline SS18 Fusions, \% & $<1$ & 0 & 0 & 0 & 0 & 0 & 100 & 0 \\
\hline MSI-High, \% & 0 & 0 & 0 & 0 & 0 & 0 & 0 & 0 \\
\hline Median TMB (mut/Mb) & 2.4 & 1.6 & 3.2 & 2.4 & 0.8 & 4.8 & 0.8 & 0.8 \\
\hline PD-L1 IHC low positive & 21 & 25 & 10 & 33 & 0 & 0 & 0 & 0 \\
\hline PD-L1 IHC high positive & 5 & 3 & 0 & 16 & 0 & 0 & 0 & 20 \\
\hline
\end{tabular}

FDCS: follicular dendritic cell sarcoma; LPS: liposarcoma; LMS: leiomyosarcoma; MPNST: malignant peripheral nerve sheath tumor; PLS: pleomorphic sarcoma; SFT: solitary fibrous tumors; SS: synovial sarcoma. 


\section{FIGURE 1.}

Longtail plots of the frequencies and types of genomic alterations in all cases of primary retroperitoneal sarcomas
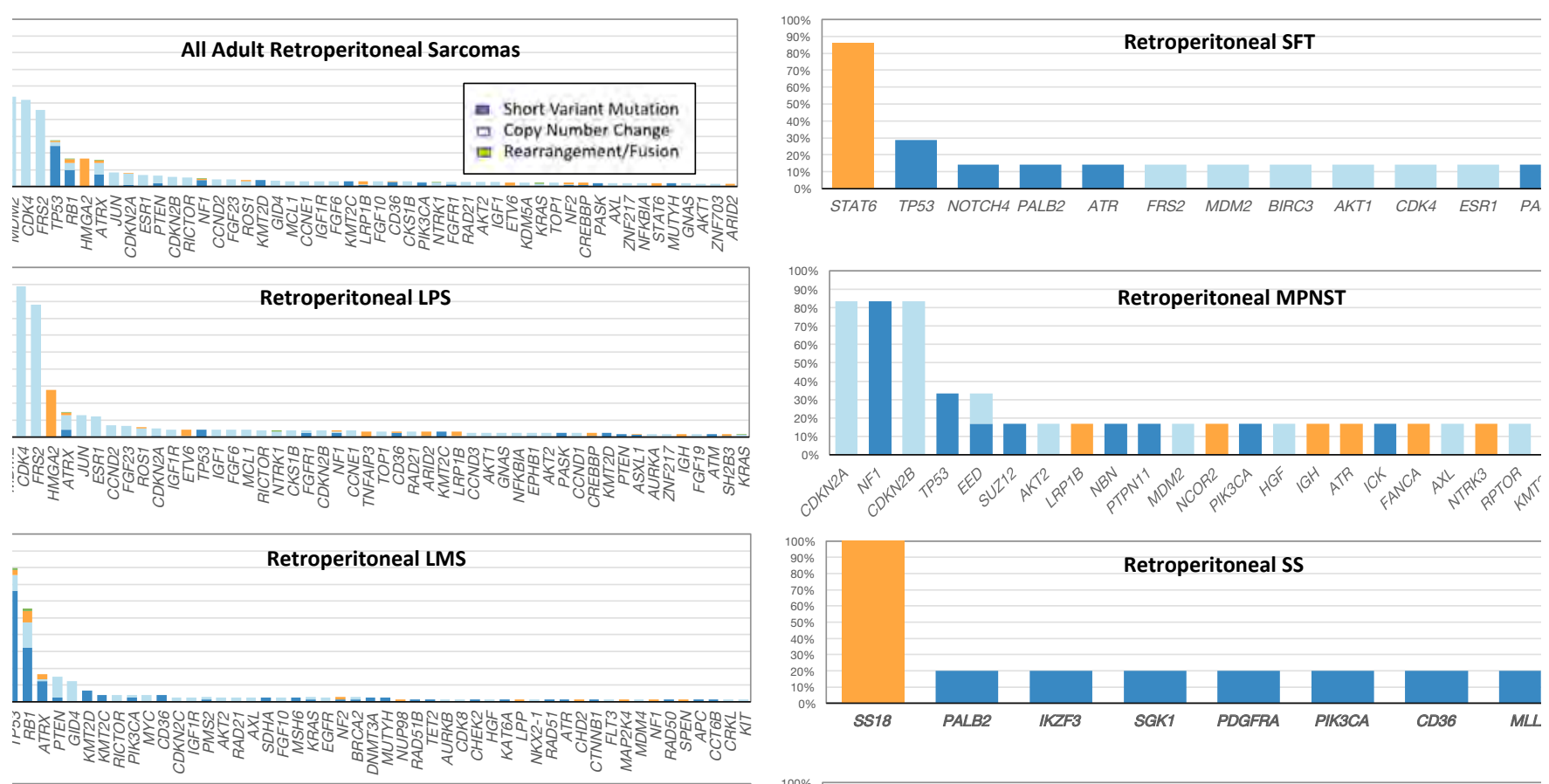

Retroperitoneal PLS

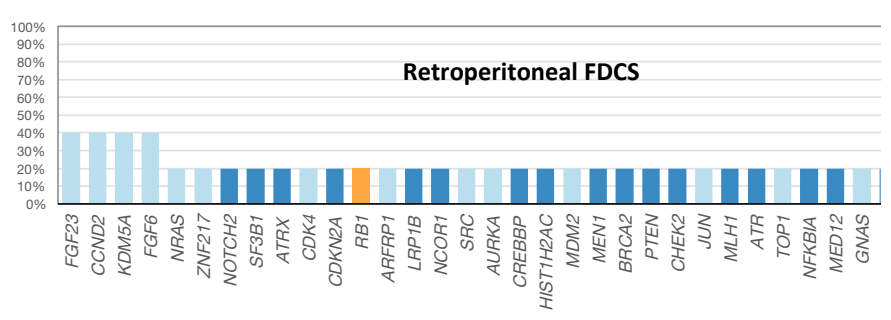

FDCS: follicular dendritic cell sarcoma ( $n=5)$; LPS: liposarcoma ( $n=155)$; LMS: leiomyosarcoma ( $n=74)$; MPNST: malignant peripheral nerve sheath tumor ( $n=6)$; PLS: pleomorphic sarcoma ( $n=44)$; SFT: solitary fibrous tumor $(n=7)$; SS: synovial sarcoma $(n=5)$

tumor featured $100 \%$ tumor cell immunohistochemical staining for PD-L1 using the Dako 22C3 assay.

\section{Discussion}

Adult RPSs are a group of rare and heterogeneous tumors marked by aggressive behavior and poor prognosis. Thus, the multidisciplinary management based on surgery, systemic therapy, and/or radiation has been the cornerstone of RPS treatment for several years. However, a great number of patients still have poor outcomes despite the implementation and continuous optimization of multimodal therapies[14]. The negative findings of the STRASS trial[15], which examined the effect of preoperative radiotherapy in RPS, suggested the idea that treatment efficacy is deeply influenced by the intrinsic biological characteristics of the different sarcomas, thus highlighting the need for a better molecular understanding of these entities. In this context, the spread of novel genomic techniques has advanced the field in this direction, also revealing several potential molecular targets and biomarkers that could offer novel opportunities in the management of these malignancies. The PALETTE trial was the landmark study to evaluate the effectiveness of a multitarget tyrosine kinase inhibitor (TKI), pazopanib, over placebo in 362 non-adipocytic soft-tissue sarcomas. The authors reported significantly prolonged median progression-free survival (PFS) in the intention-totreat cohort (4.6 versus 1.6 months) resulting in FDA approval of pazopanib in 2012 for advanced LPS refractory to systemic chemotherapy[16]. Similarly, the FDA approved eribulin for the treatment of inoperable LPS after chemotherapy, based on a phase III study that compared eribulin and dacarbazine for LPS and LMS. Although no difference was achieved in the overall 


\section{FIGURE 2.}

Pleomorphic sarcoma of the retroperitoneum in a 77-year-old woman
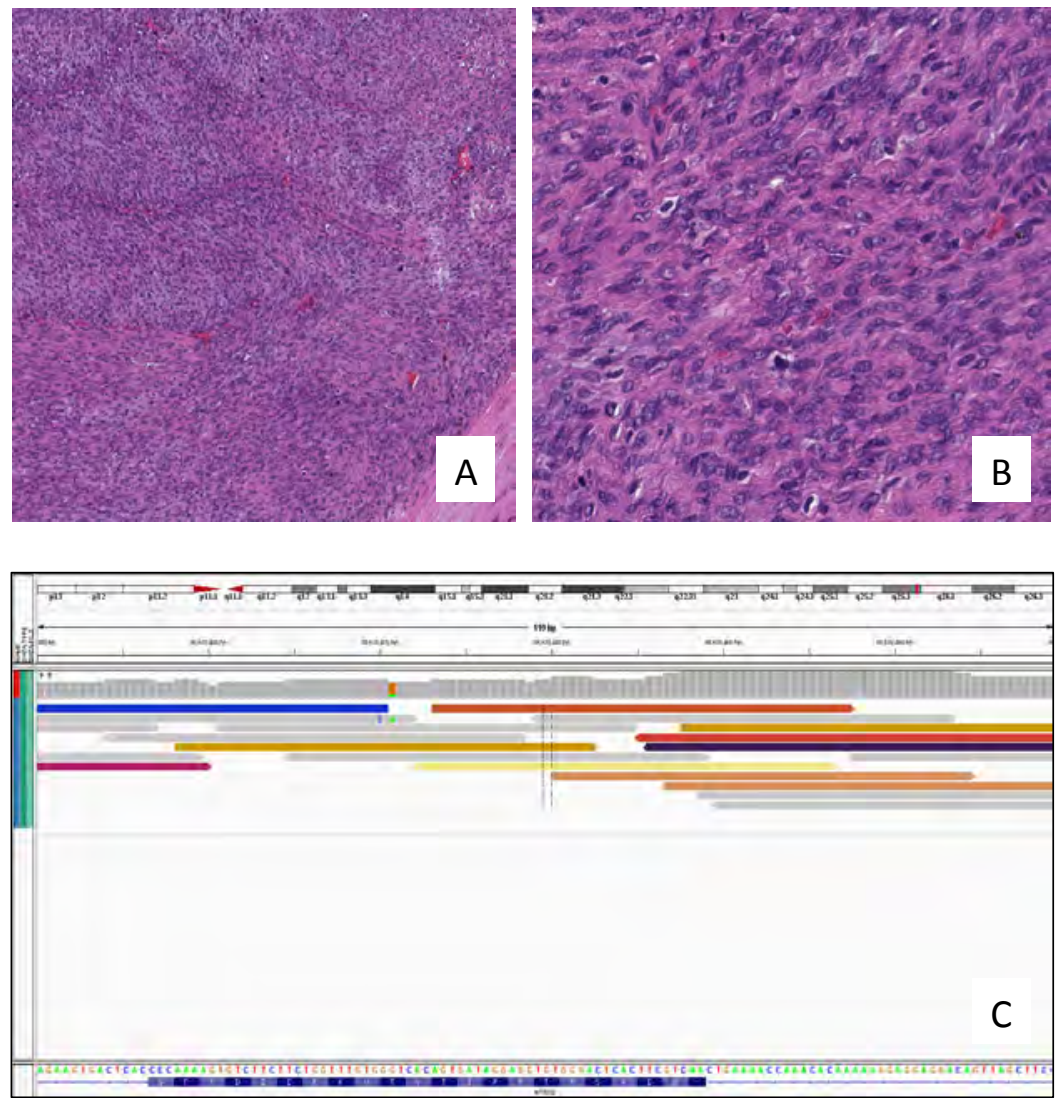

Low magnification (Figure 2A) and high magnification (Figure 2B) of a pleomorphic sarcoma. This tumor had a very high mitotic rate (20 mitoses per hpf), extensive necrosis, and stained positively for S100, SOX-10, caldesmon, and BCL2. The tumor was negative for EMA, desmin, myo-D1, CD99, CD31, CD34, pankeratins and pan melanoma markers. Comprehensive genomic profiling revealed an MSI stable tumor with intermediate TMB at 7 mutations/Mb. There was a deletion in CDKN2A/B, a short variant mutation in PBRM1 and an activating fusion in the NTRK3 gene with the STRN3 gene (5'-STRN3(ex1-3 NM_014574) (B)-NTRK3(ex12-19 NM_002530) (Figure 2C). NTRK fusions, although extremely rare, are widely distributed in solid tumors and some hematologic malignancies. This fusion has been previously described in sarcomas. Tyrosine kinases that target NTRK fusions have been approved by the regulatory agencies and include the drugs larotrectinib and entrectinib.

PFS between the 2 arms, eribulin demonstrated a statistically significant improvement in overall survival (OS) (13.5 versus 11.5 months), especially for LPS (15.6 versus 8.4 months)[17]. Following these results, our study focused on a large cohort of patients with RPS and explored targetable genomic alterations through CGP analysis. CGP analyses revealed that possible genomic targets were uncommon in our cohort of patients with RPS. In particular, RPSs were genomically stable tumors with low GA rate, low expression of PDL1 and low median TMB, suggesting low efficacy for ICI approaches. Nevertheless, genomic stratification according to histological subtypes led to the discovery of GAs that might predict patient benefit from targeted therapy testing in clinical trials. Co-amplification of $M D M 2$ and CDK4 is thought to be the main driving factor in LPS development, leading to TP53 inactivation and uncontrolled cell cycle progression[18]. MDM2 and TP53 are within the same pathway, in which MDM2 ubiquitinates TP53 and targets it for proteasomal degradation. In our cohort, LPS cases showed higher GA/tumor rate than LMS subtype, and MDM2 and CDK4 aberrations were the most frequent GAs, detected in $91 \%$ and $89 \%$ of the specimens, respectively. Similar results were recently reported by The Cancer Genome Atlas (TCGA) Research Network analysis of 206 adult sarcomas, in which the median TMB was low (1.06 mut/Mb) across the different subtypes[19]. Moreover, $M D M 2$ or CDK4 amplification was found as the highest frequent GAs in LPS subtype, as reported by other series $[20,21]$. Therefore, several clinical trials were launched testing $M D M 2 / C D K 4$ antagonists[22]. Phase I trials of MDM2 inhibitor AMG-232 alone[23] or combined with radiation therapy (NCT03217266) 


\section{FIGURE 3.}

Retroperitoneal well-differentiated liposarcoma in a 62-year-old man
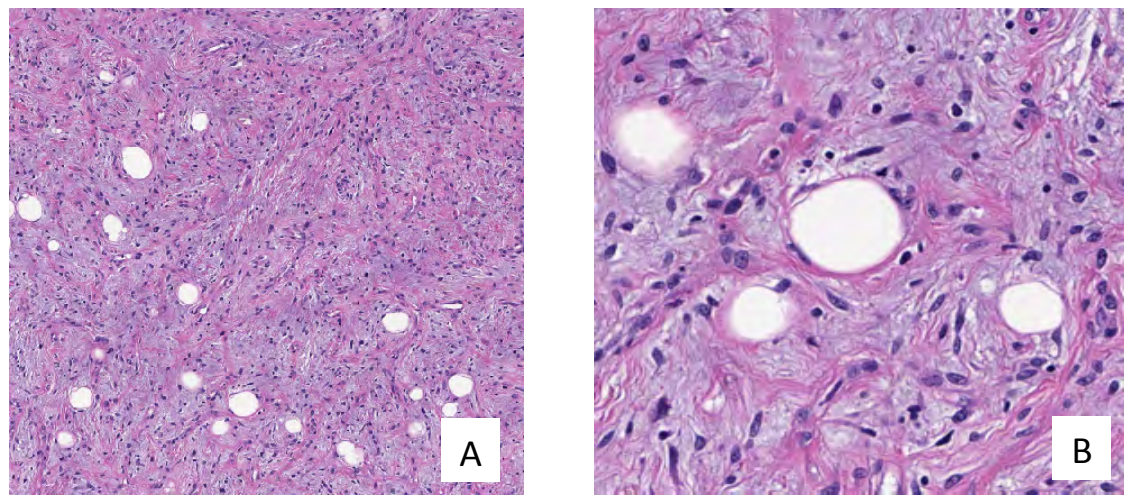

Amplification of ERBB3 (7 copies), CDK 4 (41 copies), MDM2 (90 copies), FRS2 (46 copies and ZNF217 (12 copies) $\downarrow$



\footnotetext{
Low magnification (Figure 3A) and high magnification (Figure 3B) of a retroperitoneal well-differentiated liposarcoma. This tumor was MSI stable and featured a low TMB of 2 mut/Mb. Comprehensive genomic profiling revealed (Figure 3C) amplifications of multiple genes on chromosome 12 including ERBB3 (7 copies), CDK4 (41 copies), MDM2 (90 copies), FRS2 (46 copies), and ZNF217 (12 copies). CDK4 encodes the cyclin-dependent kinase 4, which regulates the cell cycle, senescence, and apoptosis. CDK4 and its functional homolog CDK6 are activated by D-type cyclins and promote cell cycle progression by inactivating the tumor suppressor RB1. Amplification of CDK4 has been reported in lung cancer, glioblastoma, and liposarcoma.Amplification of the CDK4 and MDM2 genes, is a hallmark genetic alteration in well-differentiated liposarcoma. CDK4 amplification or activation may predict sensitivity to CDK4/6 inhibitors such as abemaciclib, palbociclib, and ribociclib.
}

showed acceptable safety in solid tumors, as well as in a cohort of dedifferentiated-LPS and SS subtypes treated with DS-3032b (NCT01877382). Similarly, encouraging results have been reported for CDK4/6 antagonists alone or in combination with doxorubicin, showing a 12 -week PFS rate between $57.2 \%$ and $66 \%$ in retroperitoneal LPS[24-26]. The second most common RPS subtype in our cohort was LMS. Our results confirmed that LMS is usually characterized by GA of tumor suppressors including TP53 (66\%) and Rb1 (32\%) $[19,20,27]$, but low frequency of GA in PTEN (12\%), which underlie potential mechanisms of resistance to ICI in LMS subtypes[28]. Conversely, MPNST subtype had the highest TMB compared with other subtypes. Nevertheless, MPNST specimens were found to be $C D K N 2 A / B$ rearranged, which is a biomarker often associated with poor prognosis and low expression of "druggable" target GA[29]. Other "targetable" gene fusions, such as $A L K$ and $R O S 1$, were rare in our population, while NTRK 1-3 gene rearrangements were mainly found in the MPNST cohort. NTRK fusions, although rare, have been described in sarcoma, and novel opportunities for patients with NTRK fusionpositive solid tumors have recently been introduced[30]. Furthermore, another GA potentially linked to targeted therapy selection is NF1 in MPNST subtype, while deletion was recently associated with $M E K$ inhibitors response[31]. In this context, novel opportunities for RPS treatment could arise from TAPUR (NCT02693535) and NCI-MATCH and the new Combo-MATCH trials testing several multi-target inhibitors according to the genomic variants expressed.

Finally, when considering PD-L1 status, $21 \%$ of RPS in our cohort had a low expression, while only $5 \%$ 


\section{FIGURE 4.}

Dedifferentiated liposarcoma of the retroperitoneum in a 74-year-old man
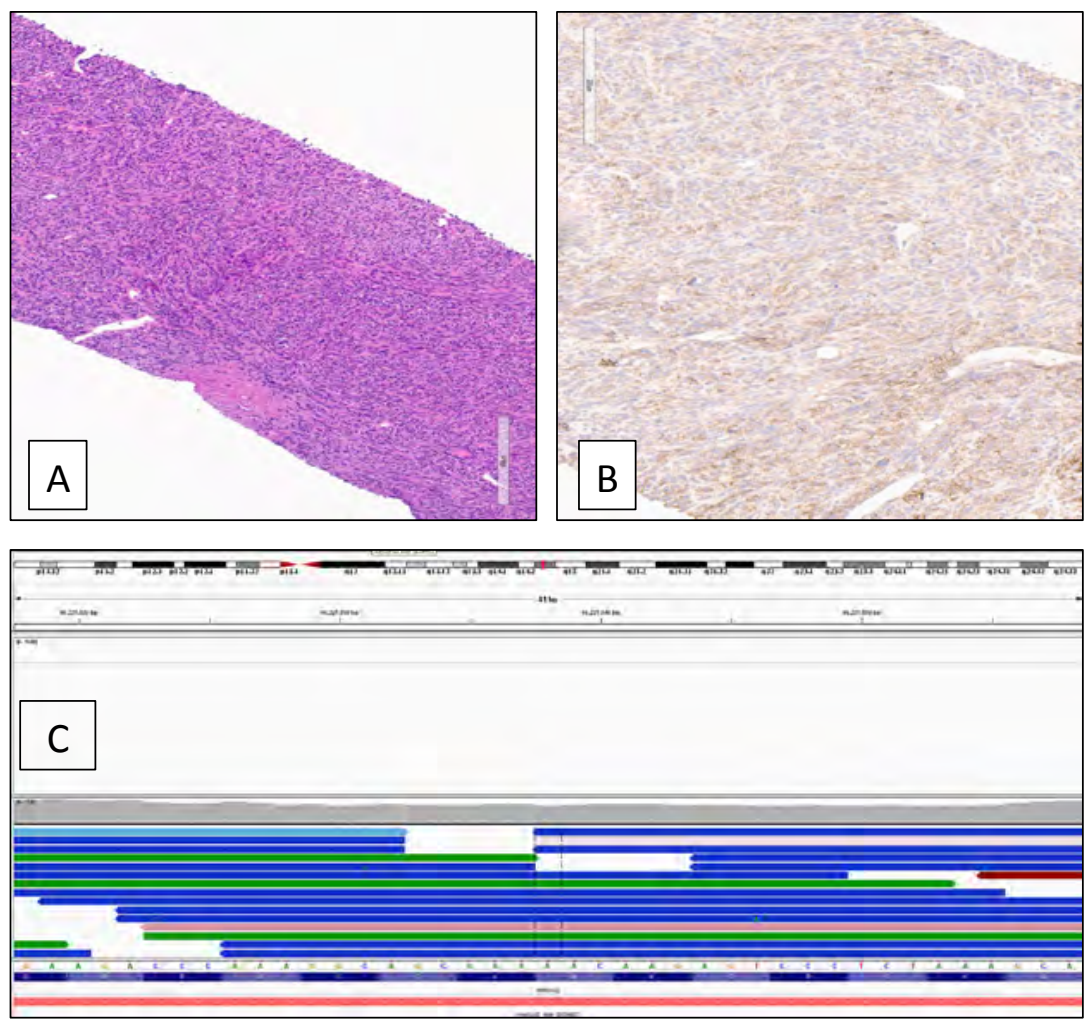

Figure 4A shows a dedifferentiated liposarcoma on hematoxylin and eosin staining at 10X magnification. Figure 4B shows diffuse positive membranous immunohistochemical staining for PD-L1 using the Dako 22C3 antibody at 10X magnification. On comprehensive genomic profiling, this MS-stable tumor has a low TMB at 3 mutations/Mb. MDM2 amplification characteristic of liposarcoma was found along with amplifications of CDK4, CCND3, FRS2 and JUN. This tumor also featured a HMGA2-TSFM fusion [Fusion:5'-HMGA2(ex1-3 NM_003483)-TSFM(ex2-6 NM_005726)] (Figure 4C). HMGA2 rearrangements and fusions have been most frequently identified in benign neoplasms such as lipomas, uterine leiomyomas, angiomyxomas, as well as in malignant tumors such as well-differentiated liposarcomas and inflammatory myofibroblastic tumors.

could be considered "high PD-L1," with PLS and FDCS subtypes associated with the highest PD-L1 expression rates. Although sarcoma is generally considered a nonimmunogenic tumor, high heterogeneity of PD-L1 expression was found across different subtypes ( $0 \%$ to $65 \%)$, suggesting that each histological subtype should be considered as a separate therapeutic challenge[32,33]. Preliminary results from the phase II SARC028 trial testing pembrolizumab for soft-tissue sarcomas reported an objective response rate of $18 \%$ and a 12 -week PFS rate of 55\%, although the subgroup analysis identified no response in the LMS cohort[34]. Conversely, combination of nivolumab plus ipilimumab has provided promising efficacy for LMS and PLS subtypes[35]. To further advance the research and understanding of RPS, it is crucial to establish joint networks to share clinical data, create centralized biobanks and prospective registries, and organize collaborative novel clinical trials. For instance, based on the increasing number of genomic alterations specifically associated with sarcoma subtypes, the design of histology- and genomic-based trials, irrespective of the tissue of origin of the sarcoma, appears a promising approach to test rational targeted agents or particular combinations. Further prospective studies are needed to confirm safety, feasibility, and efficacy of these precision oncology approaches.

This study is not devoid of limitations inherent in its nature. This was a retrospective study including available cases with a descriptive analytical approach without granular demographic and clinicopathologic features and clinical outcomes. The presence of selection and confounding biases is very likely. There was variability of tumor size, source, and viable content, and there was no central pathology review of the original tumor block; however, H\&E sections were reviewed 
by an expert pathologist. RPS included have different stages and grades which could influence the results, since the degree of tumor aggressiveness may underlie a distinct biology. We did not consider prior therapies before tumor tissue collection, which could facilitate the selection of cell clones with specific GAs and gene expression patterns. Moreover, CGP explored only a limited variety of GAs, leaving out methylomic and proteomic profile, which could possibly reveal additional important information about RPS biology. We did not evaluate circulating cell-free tumor DNA and did not pursue composite biomarker analysis.

\section{References}

1. WHO Classification of Tumours Editorial Board. WHO classification of tumours of soft tissue and bone. 5th ed. Lyon, France: IARC Press; 2020.

2. Gatta G, Capocaccia R, Botta L, Mallone S, De Angelis R, Ardanaz E, et al. Burden and centralised treatment in Europe of rare tumours: results of RARECAREnet-a population-based study. Lancet Oncol. 2017;18:1022-39. doi.org/10.1016/S1470-2045(17)30445-X.

3. Gronchi A, Miceli R, Shurell E, Eilber FC, Eilber FR, Anaya DA, et al. Outcome prediction in primary resected retroperitoneal soft tissue sarcoma: histology-specific overall survival and diseasefree survival nomograms built on major sarcoma center data sets. J Clin Oncol. 2013;31:1649-55. doi.org/10.1200/JC0.2012.44.3747.

4. Toulmonde M, Bonvalot S, Méeus P, Stoeckle E, Riou O, Isambert $\mathrm{N}$, et al. Retroperitoneal sarcomas: patterns of care at diagnosis, prognostic factors and focus on main histological subtypes: a multicenter analysis of the French Sarcoma Group. Ann Oncol.2014;25:735-42. doi.org/10.1093/annonc/mdt577.

5. Taylor BS, Barretina J, Maki RG, Antonescu CR, Singer S, Ladanyi $M$. Advances in sarcoma genomics and new therapeutic targets. Nat Rev Cancer.2011;11:541-57. doi.org/10.1038/nrc3087.

6. Pederzoli F, Bandini M, Marandino L, Ali SM, Madison R, Chung $J$, et al. Targetable gene fusions and aberrations in genitourinary oncology. Nat Rev Urol.2020;17:613-25. doi.org/10.1038/ s41585-020-00379-4.

7. Yakirevich E, Madison R, Fridman E, Mangray S, Carneiro BA, Lu $S$, et al. Comprehensive genomic profiling of adult renal sarcomas provides insight into disease biology and opportunities for targeted therapies. Eur Urol Oncol.2021;4(2):282-288. doi.org/10.1016/j. euo.2019.04.002. Epub online April 2019.

8. Frampton GM, Fichtenholtz A, Otto GA, Wang K, Downing SR, He $J$, et al. Development and validation of a clinical cancer genomic profiling test based on massively parallel DNA sequencing. Nat Biotechnol.2013;31:1023-31. doi.org/10.1038/nbt.2696.

9. He J, Abdel-Wahab 0, Nahas MK, Wang K, Rampal RK, Intlekofer AM, et al. Integrated genomic DNA/RNA profiling of hematologic malignancies in the clinical setting. Blood.2016;127:3004-14. https://doi.org/10.1182/blood-2015-08-664649.

\section{Conclusions}

Our study revealed very few potentially actionable genomic targets, suggesting that RPSs seem unlikely to respond to targeted therapies or ICI, at least based on putative molecular biomarker status. However, uncommon "targetable" kinase fusions were found depending on RPS subtypes. Further research in the different RPS subtypes is needed to explore the biology, as well as the safety and efficacy of systemic treatment regimens according to the underlying biology in attempting a precision oncology strategy.

10. Gao J, Aksoy BA, Dogrusoz U, Dresdner G, Gross B, Sumer SO, et al. Integrative analysis of complex cancer genomics and clinical profiles using the cBioPortal. Sci Signal.2013;6:pl1. doi.org/10.1126/ scisignal.2004088.

11. Cerami E, Gao J, Dogrusoz U, Gross BE, Sumer SO, Aksoy BA, et al. The cBio cancer genomics portal: an open platform for exploring multidimensional cancer genomics data. Cancer Discov.2012;2:401-4. doi.org/10.1158/2159-8290.CD-12-0095.

12. Chalmers ZR, Connelly CF, Fabrizio D, Gay L, Ali SM, Ennis R, et al. Analysis of 100,000 human cancer genomes reveals the landscape of tumor mutational burden. Genome Med.2017;9:34. doi.org/10.1186/ s13073-017-0424-2.

13. Trabucco SE, Gowen K, Maund SL, Sanford E, Fabrizio DA, Hall MJ, et al. A novel next-generation sequencing approach to detecting microsatellite instability and pan-tumor characterization of 1000 microsatellite instability-high cases in 67,000 patient samples. J Mol Diagn.2019;21:1053-66. doi.org/10.1016/j.jmoldx.2019.06.011.

14. Nazzani S, Preisser F, Bandini M, Marchioni M, Tian Z, Soulières D, et al. Surgically treated retroperitoneal sarcoma: a populationbased competing risks analysis. Eur Urol Oncol.2018;1:346-51. doi. org/10.1016/j.eu0.2018.05.008.

15. Bonvalot S, Gronchi A, Le Péchoux C, Swallow CJ, Strauss D, Meeus $P$, et al. Preoperative radiotherapy plus surgery versus surgery alone for patients with primary retroperitoneal sarcoma (EORTC-62092: STRASS): a multicentre, open-label, randomised, phase 3 trial. Lancet Oncol.2020;21:1366-77. doi.org/10.1016/S1470-2045(20)30446-0.

16. van der Graaf WTA, Blay J-Y, Chawla SP, Kim D-W, Bui-Nguyen $B$, Casali PG, et al. Pazopanib for metastatic soft-tissue sarcoma (PALETTE): a randomised, double-blind, placebo-controlled phase 3 trial. Lancet.2012;379:1879-86. doi.org/10.1016/ S0140-6736(12)60651-5.

17. Schöffski P, Chawla S, Maki RG, Italiano A, Gelderblom H, Choy E, et al. Eribulin versus dacarbazine in previously treated patients with advanced liposarcoma or leiomyosarcoma: a randomised, open-label, multicentre, phase 3 trial. Lancet.2016;387:1629-37. doi.org/10.1016/ S0140-6736(15)01283-0. 
18. Lucchesi C, Khalifa E, Laizet Y, Soubeyran I, Mathoulin-Pelissier S, Chomienne $\mathrm{C}$, et al. Targetable alterations in adult patients with soft-tissue sarcomas: insights for personalized therapy. JAMA Oncol.2018;4:1398-404. doi.org/10.1001/jamaoncol.2018.0723.

19. Abeshouse A, Adebamowo C, Adebamowo SN, Akbani R, Akeredolu T, Ally A, et al. Comprehensive and integrated genomic characterization of adult soft tissue sarcomas. Cell.2017;171:950-965. e28. doi.org/10.1016/j.cell.2017.10.014.

20. Arnaud-Coffin P, Brahmi M, Vanacker H, Eberst L, Tredan O, Attignon $V$, et al. Therapeutic relevance of molecular screening program in patients with metastatic sarcoma: analysis from the ProfiLER 01 trial. Trans/ Oncol.2020;13(12):100870. doi.org/10.1016/j.tranon.2020.100870.

21. Somaiah N, Beird HC, Barbo A, Song J, Shaw KRM, Wang W-L, et al. Targeted next generation sequencing of well-differentiated/ dedifferentiated liposarcoma reveals novel gene amplifications and mutations. Oncotarget.2018;9:19891-9. doi.org/10.18632/ oncotarget.24924.

22. Sriraman A, Dickmanns A, Najafova Z, Johnsen SA, Dobbelstein M. CDK4 inhibition diminishes p53 activation by MDM2 antagonists. Cell Death Dis.2018;9:1-16. doi.org/10.1038/s41419-018-0968-0.

23. Gluck WL, Gounder MM, Frank R, Eskens F, Blay JY, Cassier PA, et al. Phase 1 study of the MDM2 inhibitor AMG 232 in patients with advanced P53 wild-type solid tumors or multiple myeloma. Invest New Drugs.2020;38:831-43. doi.org/10.1007/s10637-019-00840-1.

24. Dickson MA, Schwartz GK, Keohan ML, D’Angelo SP, Gounder MM, Chi $P$, et al. Progression-free survival among patients with welldifferentiated or dedifferentiated liposarcoma treated with CDK4 inhibitor palbociclib: a phase 2 clinical trial. JAMA Oncol.2016;2:93740. doi.org/10.1001/jamaoncol.2016.0264.

25. Dickson MA, Tap WD, Keohan ML, D'Angelo SP, Gounder MM, Antonescu CR, et al. Phase II trial of the CDK4 inhibitor PD0332991 in patients with advanced CDK4-amplified well-differentiated or dedifferentiated liposarcoma. J Clin Oncol.2013;31:2024-8. doi. org/10.1200/JC0.2012.46.5476.

26. Infante JR, Cassier PA, Gerecitano JF, Witteveen PO, Chugh R, Ribrag $V$, et al. A Phase I study of the cyclin-dependent kinase $4 / 6$ inhibitor ribociclib (LEE011) in patients with advanced solid tumors and lymphomas. Clin Cancer Res.2016;22:5696-705. doi.org/10.1158/10780432.CCR-16-1248.
27. Chudasama P, Mughal SS, Sanders MA, Hübschmann D, Chung I, Deeg KI, et al. Integrative genomic and transcriptomic analysis of leiomyosarcoma. Nat Commun.2018;9:144. doi.org/10.1038/ s41467-017-02602-0.

28. George S, Miao D, Demetri GD, Adeegbe D, Rodig SJ, Shukla $S$, et al. Loss of PTEN is associated with resistance to anti-pd-1 checkpoint blockade therapy in metastatic uterine leiomyosarcoma. Immunity.2017;46:197-204. doi.org/10.1016/j.immuni.2017.02.001.

29. Bui NQ, Przybyl J, Trabucco SE, Frampton G, Hastie T, van de Rijn $M$, et al. A clinico-genomic analysis of soft tissue sarcoma patients reveals CDKN2A deletion as a biomarker for poor prognosis. Clin Sarcoma Res.2019;9:12. doi.org/10.1186/s13569-019-0122-5.

30. Doebele RC, Drilon A, Paz-Ares L, Siena S, Shaw AT, Farago AF, et al. Entrectinib in patients with advanced or metastatic NTRK fusionpositive solid tumours: integrated analysis of three phase 1-2 trials. Lancet Oncol.2020;21:271-82. doi.org/10.1016/S1470-2045(19)30691-6.

31. Dodd RD, Mito JK, Eward WC, Chitalia R, Sachdeva M, Ma Y, et al. NF1 deletion generates multiple subtypes of soft-tissue sarcoma that respond to MEK Inhibition. Mol Cancer Ther. 2013;12:1906-17. doi. org/10.1158/1535-7163.MCT-13-0189.

32. D'Angelo SP, Shoushtari AN, Agaram NP, Kuk D, Qin L-X, Carvajal $\mathrm{RD}$, et al. Prevalence of tumor-infiltrating lymphocytes and PD-L1 expression in the soft tissue sarcoma microenvironment. Hum Pathol. 2015;46:357-65. doi.org/10.1016/j.humpath.2014.11.001.

33. Kim JR, Moon YJ, Kwon KS, Bae JS, Wagle S, Kim KM, et al. Tumor infiltrating PD1-positive lymphocytes and the expression of pd-11 predict poor prognosis of soft tissue sarcomas. PLoS One.2013;8:e82870. doi.org/10.1371/journal.pone.0082870.

34. Tawbi HA, Burgess M, Bolejack V, Van Tine BA, Schuetze SM, Hu $J$, et al. Pembrolizumab in advanced soft-tissue sarcoma and bone sarcoma (SARC028): a multicentre, two-cohort, single-arm, openlabel, phase 2 trial. Lancet Oncol.2017;18:1493-501. doi.org/10.1016/ S1470-2045(17)30624-1.

35. D'Angelo SP, Mahoney MR, Tine BAV, Atkins J, Milhem MM, Jahagirdar BN, et al. Nivolumab with or without ipilimumab treatment for metastatic sarcoma (Alliance A091401): two openlabel, non-comparative, randomised, phase 2 trials. Lancet Oncol.2018;19:416-26. doi.org/10.1016/S1470-2045(18)30006-8. 
SUPPLEMENTARY MATERIAL 1A.

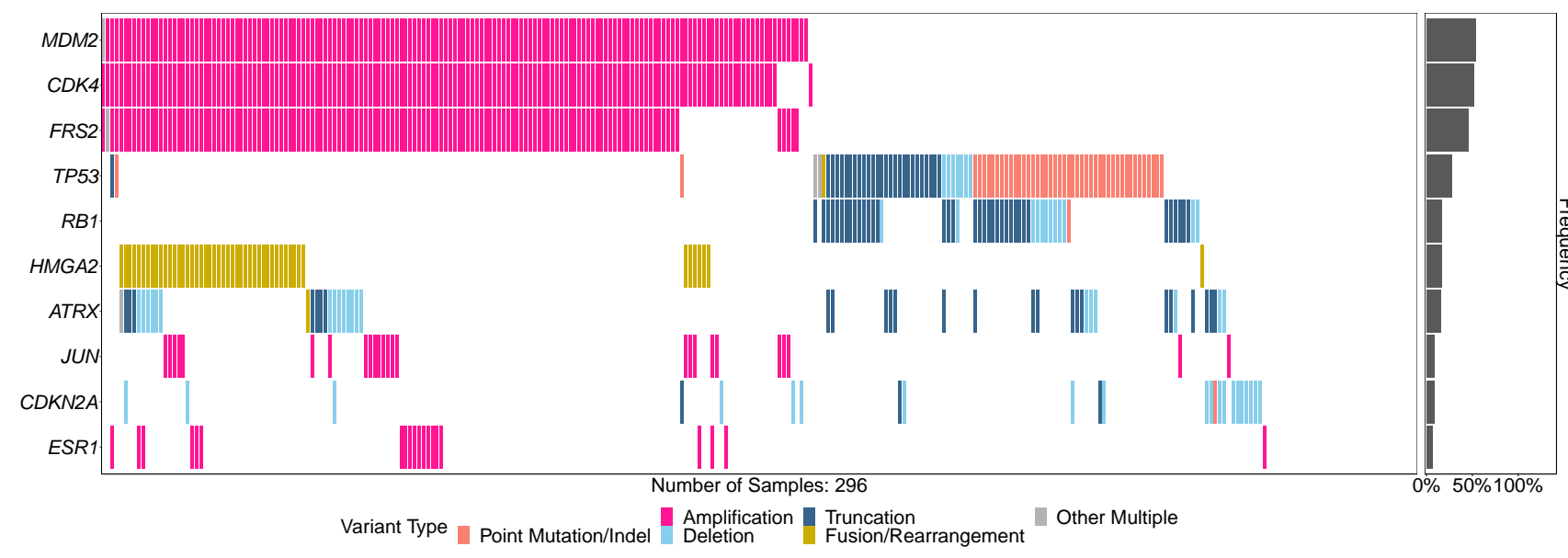

SUPPLEMENTARY MATERIAL 1B.

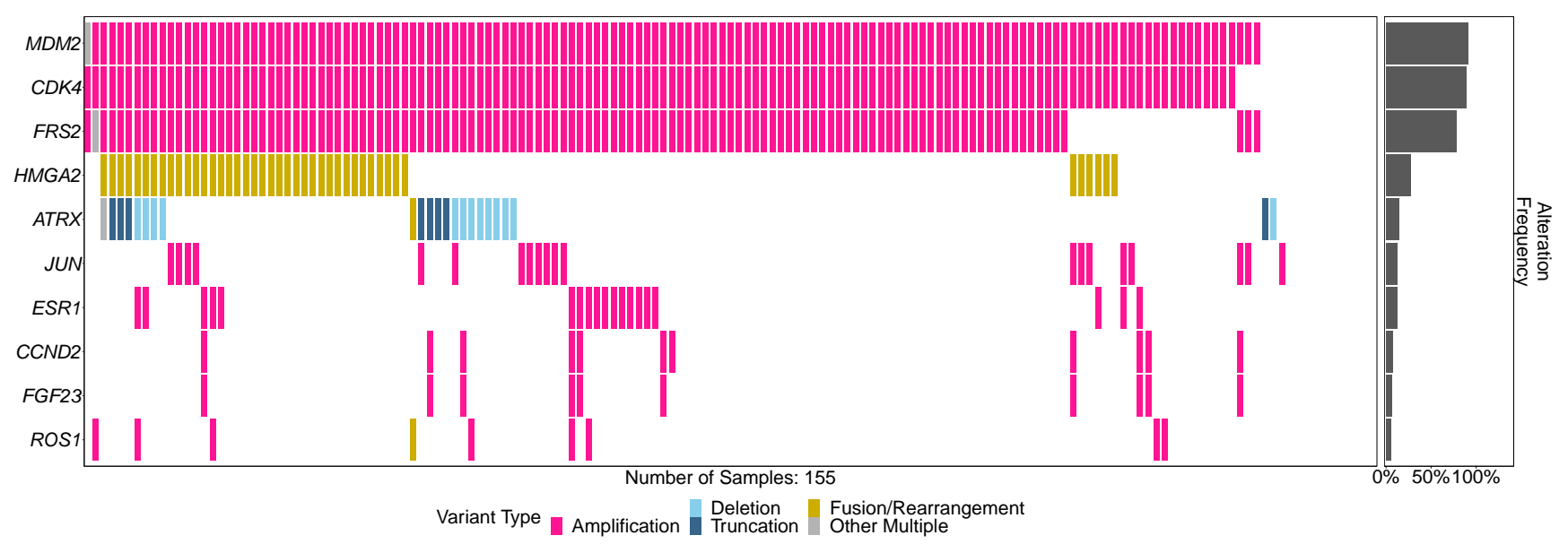

SUPPLEMENTARY MATERIAL 1C.

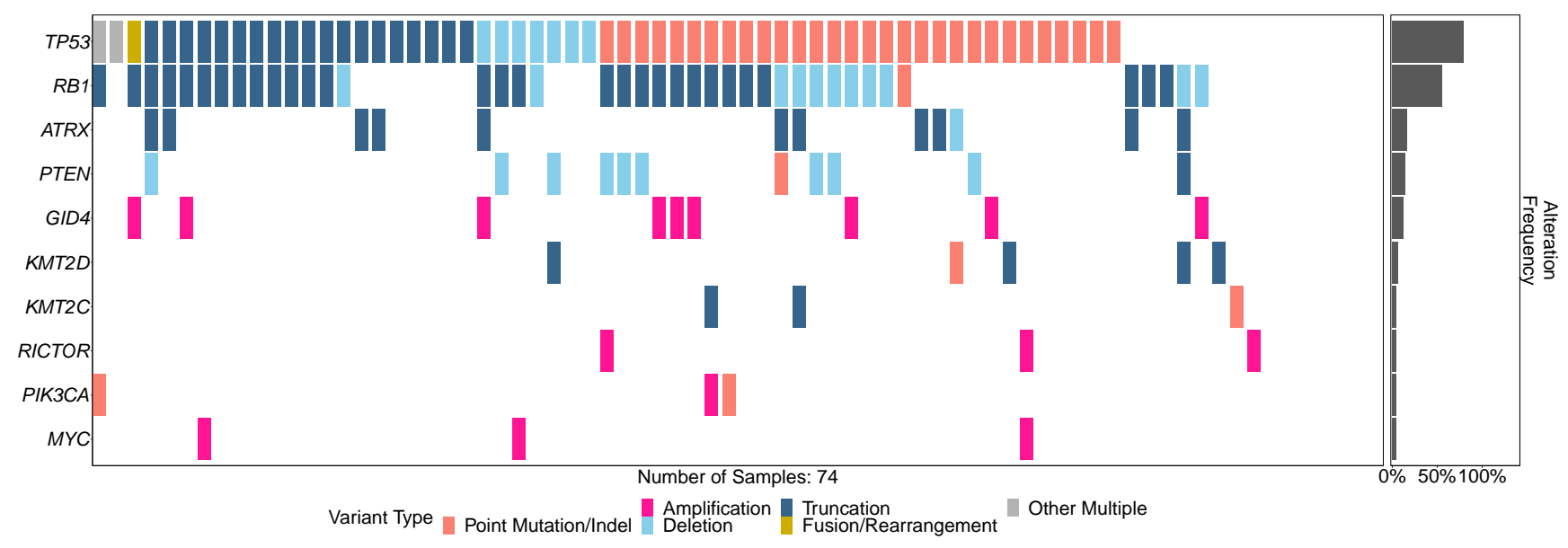


SUPPLEMENTARY MATERIAL 1D.

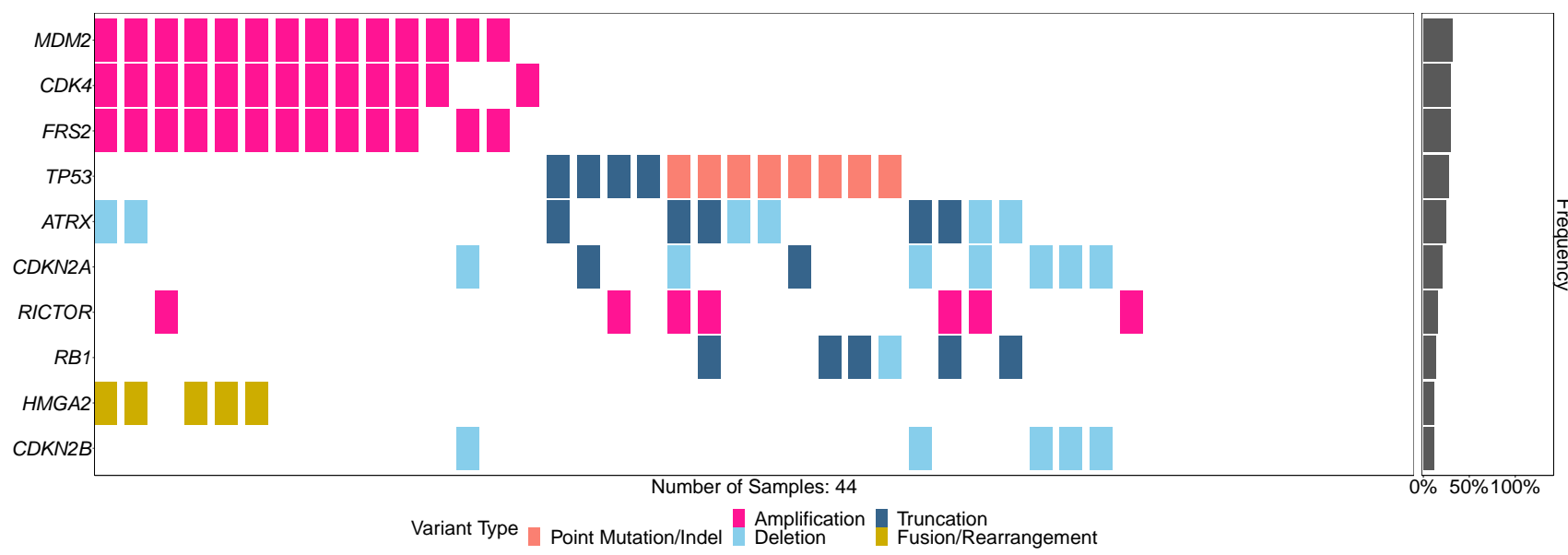

SUPPLEMENTARY MATERIAL 1E.

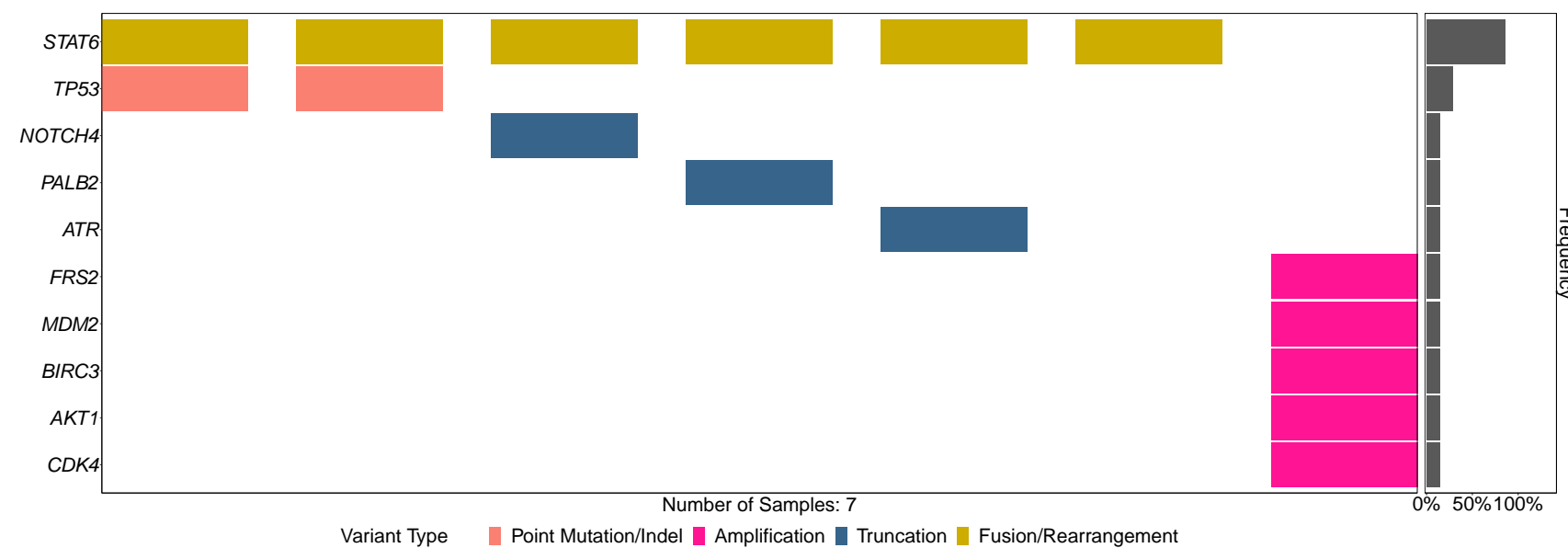

SUPPLEMENTARY MATERIAL 1C.

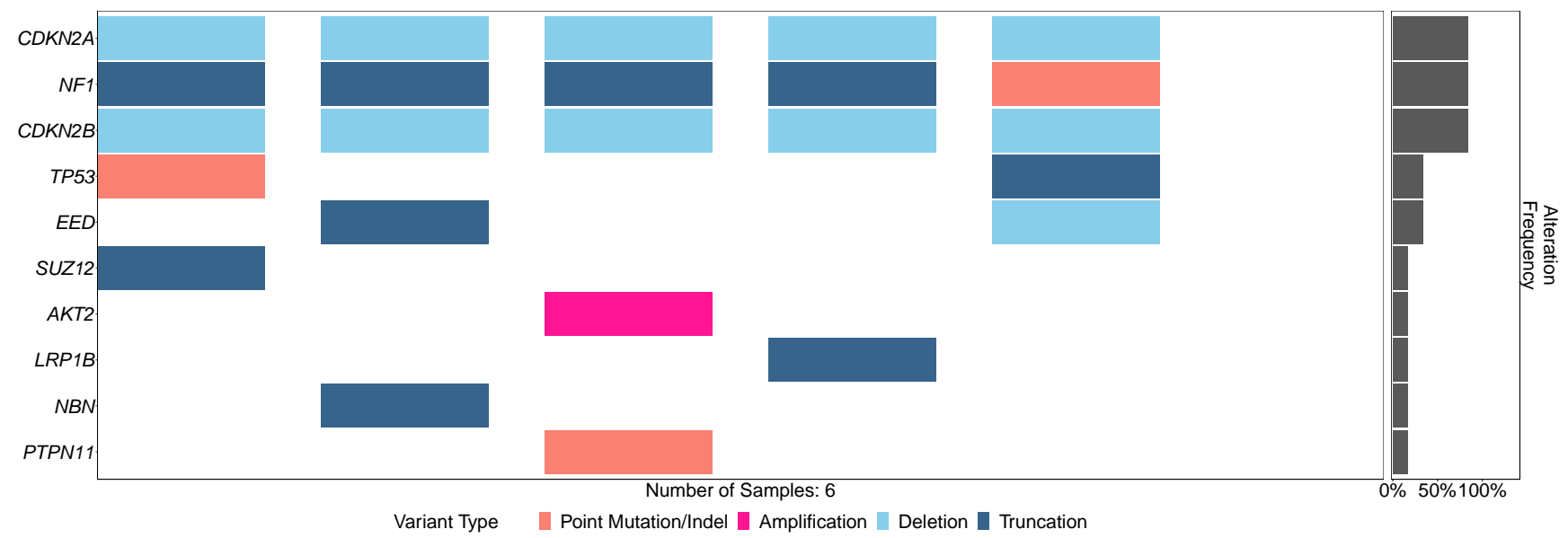


SUPPLEMENTARY MATERIAL $1 \mathrm{G}$.

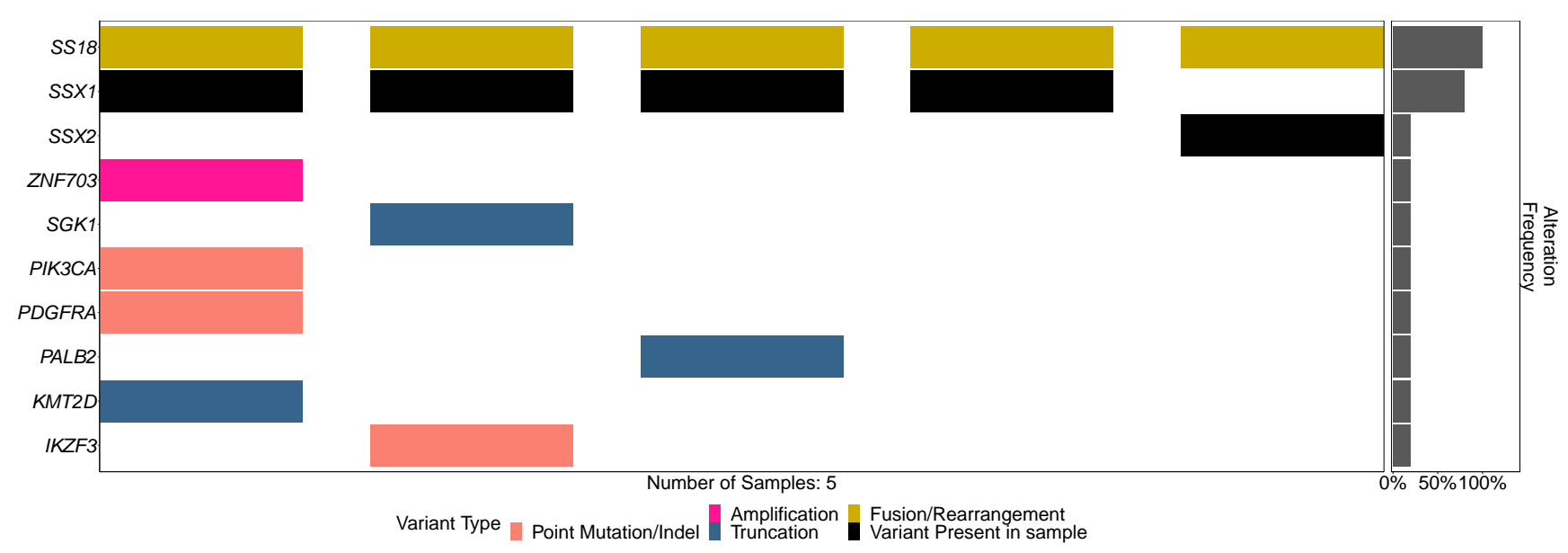

SUPPLEMENTARY MATERIAL 1H.

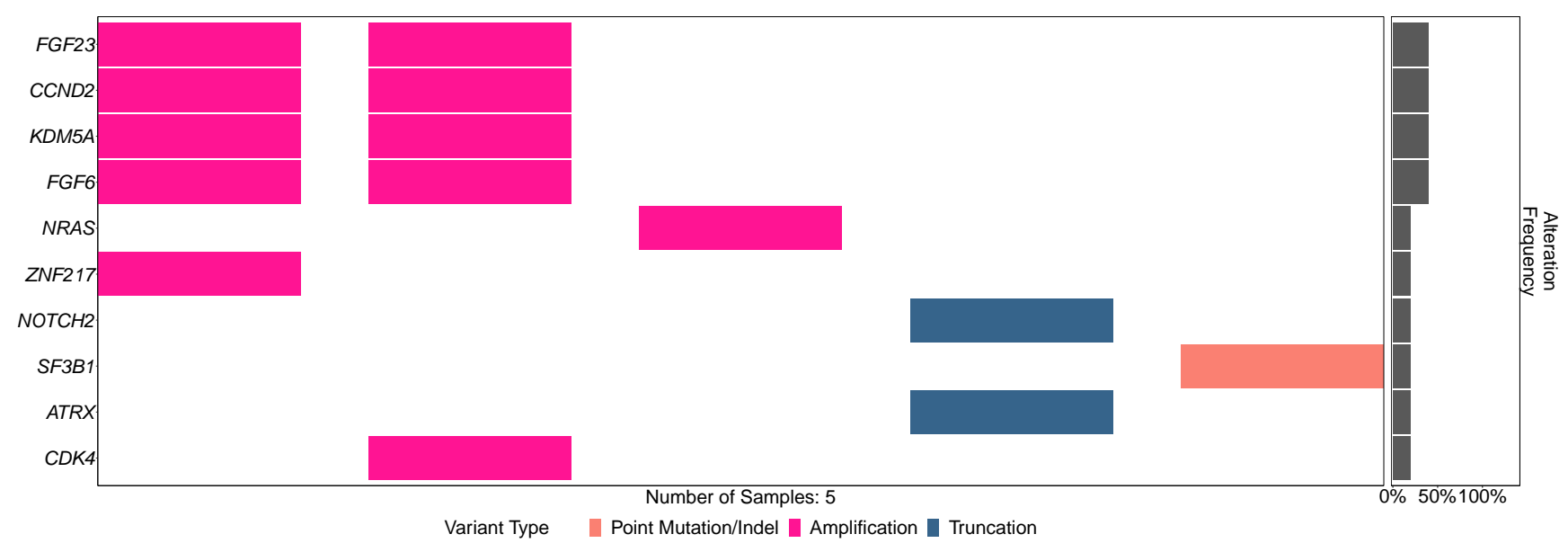

